\title{
Informating, Not Automating, the Medical Record
}

\author{
Bruce A. Friedman, M.D.
}

\begin{abstract}
The author suggests that reference should be made in the future to the need to informate and not to automate the medical record. The reason for this recommended semantic change is first to avoid the mistaken notion that the computerization of medical records will reduce the number of personnel processing medical information. Instead, personnel will shift their attention from rote clerical activities to analytic activities made possible by the creation of new data bases by computers. These new data bases, a byproduct of informating the medical record, describe work processes and lend themselves to analytic activities which will enhance quality and efficiency in hospitals. The recent availability of report generators on commercial Laboratory Information Systems (LISs) mark their transition from automating to informating systems. One example of an ad hoc report used to enhance quality and efficiency in the clinical laboratories is the throughput report.
\end{abstract}

\section{INTRODUCTION}

Hospital administrators and medical information specialists commonly refer to the automation of the medical record as an important strategic goal. By this they mean the conversion of current manual hospital information systems to computerized paper-free systems. At first glance, the use of the word automation seems appropriate to describe this evolving process, evoking a progressive image. However, these references to the automation of hospital systems often seem forced and ill-suited. Why does the term automation often evoke these unintended nuances?

The answer lies in the definition of the word automation which is a means for operating or controlling a productive process, thereby reducing human intervention to a minimum. When automating manual processes, emphasis is commonly placed on the reduction of human participation because of perceived human shortcomings or as a costsaving measure. This substitution of machines for humans is believed to increase the quality of the goods produced and reduce their cost. The prerequisite, therefore, of the automation of processes is reduced human participation in the activity being automated.

From the Department of Pathology, University of Michigan Medical School, Ann Arbor, Michigan 48109-0602. 
Some authors indicate that the automation of hospital information systems actually accomplishes this goal of reducing human participation: "appropriate information technology should eliminate $1 \frac{1 / 2}{2}$ hours of clerical activity per nursing service employee per shift." Notice, however, that specific reference is made in this quotation to a reduction in clerical activity and not to the total participation of nursing personnel in information processing.

\section{INFORMATING WORK PROCESSES IN HOSPITALS}

I believe that it is unlikely that the automation of the medical record will result in any net decrease in the information processing by health care workers. Rather it will cause a shift of work away from repetitive data entry tasks, often referred to as clerical activities, and toward an increased amount of analytic activity. Zuboff comments specifically on this shift in activities by workers as they begin to interact with information systems:

\footnotetext{
As time frames become more immediate, as more sectors of data can be integrated, as software helps limit inaccuracies, as data entry and access becomes more widely distributed, and as programmed logic becomes more comprehensible and flexible, the surrounding life-world of the organization comes to be more comprehensively reflected in a dynamic, fluid electronic text. New methods of automating this textualization process, such as ... sophisticated automated data-entry devices . . . mean that fewer people will be needed to accomplish routine transactions in conjunction with the machine system. ${ }^{2}$
}

Zuboff also observes that information technology provides the means for facilitating processes and providing more continuity and control. In this context, she emphasizes that this same information technology will generate information about the underlying processes through which an organization accomplishes its work. It is this latter ability to provide "a deeper level of transparency to activities that had been either partially or completely opaque in the past" which causes information systems to transcend the traditional logic and definition of automation. ${ }^{3}$

Zuboff has coined the verb "to informate" to capture the idea that processes or activities can be unmasked by information systems when a technology informates as well as automates. One of the major goals during the computerization of medical records is to take advantage of this specific capability of computers. These new insights can be used to increase the quality and efficiency of work. Because of the described shortcomings inherent in the term automation, I therefore urge that health care workers begin to refer to the process of informating rather than automating the medical record.

\section{JUSTIFICATION FOR THE SEMANTIC CHANGE}

Semantic modifications do not come easily and never without compelling reasons for the change. What are the benefits if the change advocated here is adopted? First, it will be easier to discard the widely-accepted and false idea that the implementation of computerized information systems will inexorably result in a reduction in the number of personnel processing medical information. Remember that the word automation implies a reduction in human participation in the process automated. Secondly, it will become more 
obvious through the use of the new word that a byproduct of the computerization of the medical record will be new data bases describing work processes.

Wyszewianski and colleagues point out that these new data bases and the analytic opportunities presented by them will allow the development of management and operational controls to enhance production and clinical efficiency as well as quality. ${ }^{4}$ Many of the analytic tasks made possible by these new data bases will be assigned to those very workers who have been emancipated from the tedious data entry jobs by the informating systems. This is the basis for my belief that informating the medical record will not result in any net decrease in the total worker participation in hospital information processing.

Zuboff uses the term "textualization" which requires further explanation because it sheds light on the evolving role of knowledge workers. Computers serve to introduce an informating technology into the work place. As the goal of informating the medical record is pursued in hospitals and processes not previously documented become informated, the computer technology can be said to have textualized the environment. That is, the "electronic text becomes a new medium in which events are both observed and enacted.",5

Workers in such a newly textualized environment are offered the opportunity to manage and add value to the information product of their jobs because of the new electronic medium. ${ }^{6}$ Reference has been made in a previous article to the way in which information systems add value to the data processed and that this added value can be calibrated by the quality and efficiency gains introduced by such systems. ${ }^{7}$

\section{THE ANALYTIC COMPONENT OF INFORMATION SYSTEMS}

The process of informating the medical record defines new opportunities for the enhancement of both the quality and efficiency of health care delivery through the creation of new data bases describing work processes. A shorthand way of describing these new quality and efficiency capacities of systems is to refer to their analytic capabilities.

Hospital information systems, according to Griffith, provide the means for capturing, transmitting, storing, and, analyzing information. ${ }^{8}$ The capturing aspect of hospital systems consists of their ability to improve the accuracy and speed with which transactions between patient care units and systems are recorded and communicated. The analytic aspect of systems in the early stages of system development consists of the provision of current performance data which can be compared with expectations or performance criteria using software tools. These analytic capabilities can be enhanced using long-term archival files which provide the opportunity for a more thorough understanding of performance and causality.

Griffith describes the evolving analytic capabilities of information systems as a continuum. The process starts with the analysis of the work processes themselves, supported by archival information and performance criteria which define optimal performance from a quality and efficiency perspective. Enhancements made to these work processes depend on human intervention guided by the results of this analytic activity. The next step in the continuum is the heuristic phase during which information systems, with or without human collaboration, assist in making changes to achieve optimal performance. $^{9}$

In summary, the automation of the medical record includes tasks such as transaction 
processing with particular emphasis on order entry-result reporting functions and charge capture. Informating the medical record goes beyond this initial automation stage and conveys the idea of reaching for the more sophisticated application plateau of enhanced analytic capabilities with increased opportunities for quality and efficiency enhancements.

\section{USING THE LABORATORY INFORMATION SYSTEM FOR ANALYSIS OF WORK PROCESSES}

The Laboratory Information System (LIS) is one of the oldest and most widelyimplemented medical systems in hospitals. The term medical system is used here to mean an information system intended to process information which facilitates direct patient care and increases the efficiency and quality of work physicians and nurses. This is in contrast to administrative systems which encompass applications such as financial and admission/discharge/transfer. Older LISs were designed primarily to create hardcopy reports of test results for individual patients and were not capable of producing sophisticated management reports requiring inter-patient comparisons.

LISs now available in the commercial market come equipped with data base management systems (DBMS), often referred to as report generators, which can create a wide variety of $a d$ hoc reports. LIS report generators can be used to address concerns in four distinct areas: (1) quality issues (2) management (efficiency) issues; (3) data retrieval to satisfy governmental and accreditation agency requirements; (4) data retrieval for research purposes.

These report generators provide clinical laboratory personnel with the analytic capability to informate their specific component of the medical record. The availability of this feature thus serves as a benchmark for the transition of LISs from automating systems to informating systems. Friedman has commented specifically on the impact of new features of commercial LISs such as report generators on quality assurance programs. ${ }^{10}$

Let us examine one example of an ad hoc report generated by a LIS and its report generator to understand the power the system as an analytic tool and its potential impact on quality and efficiency. A report can now be easily developed by the LIS on a periodic basis showing the intra-laboratory turnaround time by hour for routinely ordered tests (throughput report). The analysis of test turnaround time is useful in assessing the efficiency of clinical laboratory operations and staffing patterns. The mean length of testordering cycles in a hospital also has an obvious relationship to a patient's hospital length-of-stay. For hospital patients such as those in critical care units, test turnaround time has significant quality of care implications.

Turnaround time by test can be further categorized by routine and stat test requests. A prolonged test turnaround time may be the result of one or more of the following: (1) inefficient intra-laboratory work flow; (2) an increased volume of specimen flow into the laboratories; (3) the mix of routine and stat requests submitted to the laboratory; (4) the type of automated instruments used in the laboratory; or (5) laboratory personnel staffing patterns.

A report generator can be used to analyze the origin of specimens submitted to the clinical laboratories if turnaround time is inadequate and resources are not available to staff the laboratories for peak workload. An alternative solution may be to stagger inpa- 
tient blood drawing sweeps by phlebotomists or the scheduling of those clinics which account for heavy laboratory test activity.

Laboratory personnel will undoubtedly come to regard the LIS report generator as an important analytic tool for enhancing the quality and efficiency of laboratory operations. The same analytic power can also be used to review the relationship between abnormal test results and surgical pathology diagnoses with patient discharge diagnoses or surgical procedures performed during the hospital stay. This latter capability presupposes the ability to merge discharge diagnosis and procedure codes with the laboratory data base.

It has always been assumed that computers would have a major effect on the nature of work. Only recently have information theorists begun to understand the nature of this process. It is appropriate to develop a new vocabulary to mark this transition to this new understanding and encompass some new subtleties of expression.

\section{REFERENCES}

1. Mowry, M.M., and Korpman, R.A., Automated information systems in quality assurance. Nurs. Econ. $5: 237-244,1987$.

2. Zuboff, S., In the Age of the Smart Machine. The Future of Work and Power, Basic Books, Inc., New York, 1988, p. 237-244.

3. Zuboff, S., In the Age of the Smart Machine. The Future of Work and Power, Basic Books, Inc., New York, 1988, p. 172.

4. Wyszewisnski, L., Thomas, J.W., and Friedman, B.A., Case-based payment and the control of quality and efficiency in hospitals. Inquiry 24:17-25, 1987.

5. Zuboff, S., In the Age of the Smart Machine. The Future of Work and Power, Basic Books, Inc., New York, 1988, pp. 9-10.

6. Zuboff, S., In the Age of the Smart Machine. The Future of Work and Power, Basic Books, Inc., New York, 1988, p. 216.

7. Friedman, B.A., Laboratory information systems and the competency trap. In Greenes, R.A., ed., Proceedings of the Twelfth Annual Symposium on Computer Applications in Medical Care, IEEE Computer Society Press, New York, 1988, pp. 659-662.

8. Griffith, J.R., The Well-Managed Community Hospital, Health Administration Press, Ann Arbor, 1987, p. 332.

9. Griffith, J.R., The Well-Managed Community Hospital, Health Administration Press, Ann Arbor, 1987, p. 329.

10. Friedman, B.A., The impact of new features of laboratory information systems on quality assurance in anatomic pathology [editorial]. Arch. Pathol. Lab. Med. 112:1189-1191, 1988. 